\title{
CircRNA-0044073 is upregulated in atherosclerosis and increases the proliferation and invasion of cells by targeting miR-107
}

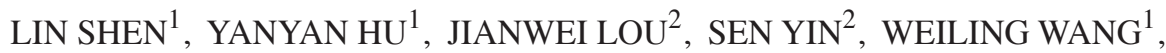 \\ YUANYAN WANG ${ }^{1}$, YONG XIA ${ }^{1}$ and WEI WU ${ }^{2}$ \\ ${ }^{1}$ Department of Geriatrics, Key Laboratory of Cardiovascular Proteomics of Shandong Province, \\ Qi-Lu Hospital of Shandong University; ${ }^{2}$ Department of Neurology, Qi-Lu Hospital of Shandong University and \\ Brain Science Research Institute, Shandong University, Jinan, Shandong 250012, P.R. China
}

Received April 15,2018; Accepted September 13, 2018

DOI: $10.3892 / \mathrm{mmr} .2019 .10011$

\begin{abstract}
Circular RNAs (circRNAs) are endogenous non-coding RNAs implicated in atherosclerosis. The aim of the present study was to explore the function of circRNA-0044073 in atherosclerosis. Reverse transcription quantitative polymerase chain reaction assays were used to measure the expression levels of circRNA-0044073, microRNA (miRNA/miR)-107, janus kinase 1 (JAK1), signal transducer and activator of transcription 3 (STAT3), B-cell lymphoma 2 (Bcl-2) and v-myc avian myelocytomatosis viral oncogene homolog (c-myc) in in blood cells from patients with atherosclerosis. RNA pull-down and luciferase reporter assays were then used to determine the association between circRNA and miR expression, and $\mathrm{miR}$ and gene expression, respectively. Matrigel invasion assay and flow cytometry were used to analyze cell invasion and cell cycle. Western blot analysis and ELISA were used to evaluate the expression levels of proteins. It was identified that the expression of circRNA-0044073 was upregulated and the expression of miR-107 was downregulated in atherosclerotic blood cells. Overexpression of circRNA-0044073 promoted the proliferation of human vascular smooth muscle cells (HUVSMCs) and human vascular endothelial cells (HUVECs), while overexpression of miR-107 inhibited their proliferation. In addition, circRNA-0044073 suppressed the levels of miR-107 via a sponge mechanism. Lipopolysaccharide (LPS) affected the proliferation of HUVSMCs and HUVECs, and also resulted in changes in circRNA-0044073 expression levels. CircRNA-0044073 promoted the proliferation and invasion of HUVSMCs and HUVECs in spite of the opposite effect observed with LPS treatment. The JAK/STAT signaling
\end{abstract}

Correspondence to: Dr Wei Wu, Department of Neurology, Qi-Lu Hospital of Shandong University and Brain Science Research Institute, Shandong University, 107 Wenhuaxi Road, Jinan, Shandong 250012, P.R. China

E-mail:wuwei7172@126.com

Key words: circular RNA-0044073, microRNA-107, janus kinase 1, signal transducer and activator of transcription 3, atherosclerosis pathway was activated in patients with atherosclerosis. CircRNA-0044073 favored the activation of the JAK/STAT signaling pathway and inflammation in HUVSMCs and HUVECs. These data indicate that circRNA-0044073 is upregulated in atherosclerosis and promotes the proliferation and invasion of cells by targeting miR-107 and activating the JAK/STAT signaling pathway, potentially offering a target for novel treatment strategies against atherosclerosis.

\section{Introduction}

Atherosclerosis is a chronic inflammatory disorder of the arterial vessel walls characterized by lipid deposition and fibrous cap formation in the arterial intima. Atherosclerosis contributes to cardiovascular disease (CVD), a major cause of morbidity and mortality in Western countries. A total of \% 80 million Americans have one or more forms of CVD (1). Growing evidence suggests that dysfunction of endothelial cells (ECs) and vascular smooth muscle cells (VSMC) is critical in the formation of atherosclerosis. Abnormal proliferation and migration of these cells contribute to the progression of atherosclerosis (2-4). VSMCs migrate and proliferate to form a stabilizing fibrous cap that encapsulates atherosclerotic plaques (5). During the initiation and development of inflammation, ECs and VSMCs produce numerous types of cytokines, including tumor necrosis factors (TNF), interleukin (IL), adhesion molecules, interferon, and adventitium-derived relaxing factors that are implicated in atherosclerosis (6-9).

Circular RNAs (circRNAs) are evolutionarily conserved non-coding RNAs produced by the scrambling of exons at the time of splicing. They are primarily produced in the brain and are present inside the cell. Previous data have suggested that the circRNA antisense non-coding RNA in the INK locus modulates ribosomal RNA maturation and atherosclerosis in humans, indicating that circularization of long non-coding RNAs may alter RNA function and therefore protect against disease (10). The great potential of circRNAs as biomarkers for the early detection of cardiovascular diseases has been highlighted in several patents and previous studies (11). Certain circRNAs, including circRNA-0003575 and circRNA-000595, are significantly associated with atherosclerosis $(12,13)$. Furthermore, the role of circRNAs in atherosclerosis is associated with the 
regulation of the physiology of ECs and smooth muscle cells (SMCs) (10). MicroRNAs (miRNAs) are a group of highly conserved small non-coding RNA molecules measuring 18-22 nucleotides in length. By binding to the 3'-untranslated region of their target mRNAs, miRNAs regulate expression levels of downstream genes $(14,15)$. In addition to participating in regulating cell-cycle and cell proliferation $(16,17)$, miRNAs are associated with inflammatory processes involved in atherosclerosis (18). miR-107 is implicated in the pathogenesis of diseases, including renal disease, Alzheimer's disease, gastric cancer, colon cancer and hepatocellular carcinoma (19-22). Previous studies suggested that miR-107 is a regulator of atherosclerosis $(23,24)$. In addition, binding of miR-107 to the Clock circadian rhythm gene resulted in the deregulation of the circadian rhythm of the cells $(25,26)$. However, the exact mechanism is largely unknown.

Certain circRNAs have been described as regulatory transcripts as they behave like sponges to bind miRNAs. For example, a previous study examining the role of circular RNA-7 demonstrated that the circRNA was an inhibitor of miR-7, an miRNA known to regulate various diseases including, cancer, neurodegenerative diseases, diabetes and atherosclerosis (27). In this context, the present study aimed to investigate the role of circRNA in the regulation of atherosclerosis and to understand the underlying mechanism associated with miRNAs and signaling pathways.

\section{Materials and methods}

Patients and samples. Human blood cells were collected from 20 patients with atherosclerosis and healthy controls at the Qi-Lu Hospital of Shandong University (Jinan, China). In total, 13 males and seven females (age range, $48-68$ years) were enrolled in the study. Following sonographic examination, the patients with a carotid intima-media thickness $\geq 1.3 \mathrm{~mm}$ were considered to exhibit an atherosclerotic plaque. Informed consent was obtained from all patients and ethical approval was granted by The Ethics Committee of the Qi-Lu Hospital of Shandong University.

Cell culture and treatment. All reagents for cell culture were obtained from Invitrogen; Thermo Fisher Scientific, Inc. (Waltham, MA, USA). Human umbilical vein endothelial cells (HUVECs) were purchased from the American Type Culture Collection (ATCC, Manassas, VA, USA), and human umbilical vein smooth muscle cells (HUVSMCs) were purchased from iXCells Biotechnologies (San Diego, CA, USA). HUVECs and HUVSMCs cells were maintained in minimum essential medium-L-glutamine (Sigma-Aldrich; Merck KGaA, Darmstadt, Germany), smooth muscle cell growth medium and RPMI-1640 medium (Gibco; Thermo Fisher Scientific, Inc.). The medium was supplemented with $10 \%$ fetal bovine serum (FBS; Gibco; Thermo Fisher Scientific, Inc.) and 1\% penicillin-streptomycin-amphotericin. The cells were maintained in a humidified incubator with $5 \% \mathrm{CO}_{2}$ at $37^{\circ} \mathrm{C}$. The culture medium was changed twice weekly. HUVECs and HUVSMCs were sub-cultured subsequent to reaching $80 \%$ confluency. For the following assay, the cells initially received either $100 \mathrm{ng} / \mathrm{ml}$ lipopolysaccharide (LPS) or vehicle. The template DNA of circRNA-0044073 was synthesized and then inserted into the multiple cloning site in the pAd-Track-cmv vector (Addgene, Inc., Cambridge, MA, USA). A total of $500 \mathrm{ng}$ of plasmid DNA was subsequently transfected into HUVECs or HUVSMCs in 24-well plates using Lipofectamine ${ }^{\circledR} 2000$ (Invitrogen; Thermo Fisher Scientific, Inc.) to increase circRNA-0044073 expression, whereas, the empty vector was transfected as the negative control (NC). Following $48 \mathrm{~h}$ of incubation, the transfected cells were used in the following experiments.

Reverse transcription quantitative polymerase chain reaction $(R T-q P C R)$. Total RNA was isolated from cells using TRIzol ${ }^{\circledR}$ reagent (Thermo Fisher Scientific, Inc.) and was reverse transcribed using the Bestar qPCR RT kit (DBI Bioscience, Ludwigshafen, Germany) at $65^{\circ} \mathrm{C}$ for $5 \mathrm{~min}$. qPCR amplification was performed in triplicate using DBI Bestar ${ }^{\circledR}$ SybrGreen qPCR MasterMix (DBI Bioscience, Ludwigshafen, Germany) in a total volume of $20 \mu \mathrm{l}$ containing $2 \mu \mathrm{l}$ each cDNA. The amplification protocol consisted of initial denaturation for $2 \mathrm{~min}$ at $94^{\circ} \mathrm{C}$, followed by 40 cycles of denaturation $\left(94^{\circ} \mathrm{C}\right.$ for $20 \mathrm{sec}$ ), annealing for $20 \mathrm{sec}$ at $58^{\circ} \mathrm{C}$ and elongation for $20 \mathrm{sec}$ at $72^{\circ} \mathrm{C}$. The primers for circRNA-0044073, miR-107, U6, janus kinase 1 (JAK1), signal transducer and activator of transcription 3 (STAT3), B-cell lymphoma 2 (Bcl-2), v-myc avian myelocytomatosis viral oncogene homolog (c-myc) and GAPDH are summarized in Table I. Using GAPDH or U6 as endogenous control genes, the relative transcript levels were calculated using the $2^{-\Delta \Delta \mathrm{Cq}}$ quantification method (28).

Cell viability assay. Cell viabilities were assessed by the Cell Counting Kit-8 (Dojindo Molecular Technologies, Inc., Kumamoto, Japan). In brief, cells were added into a 96-well plate at a density of $2 \times 10^{3}$ cells/well and incubated overnight at $37^{\circ} \mathrm{C}$. Then, LPS was added to each well and incubated at $37^{\circ} \mathrm{C}$ for 24,48 and $72 \mathrm{~h}$ at the final concentration of $100 \mathrm{ng} / \mathrm{ml}$. Thereafter, $10 \mu \mathrm{l}$ CCK- 8 solution was added into each well and incubated for $2 \mathrm{~h}$ at $37^{\circ} \mathrm{C}$. The absorbance was detected at $450 \mathrm{~nm}$ with the microplate reader. Optical density was defined as the relative number of viable cells. The assay was performed in triplicate.

Western blot analysis. Following treatments with LPS for $24 \mathrm{~h}$, cells were washed with PBS and subsequently harvested. Total cellular proteins were extracted with radioimmunoprecipitation assay lysis buffer containing $1 \%$ phenylmethanesulfonyl fluoride (Sigma-Aldrich; Merck KGaA). Protein concentrations were determined with a BCA assay (Bio-Rad Laboratories, Inc., Hercules, CA, USA). Subsequent to denaturation, a total of $20 \mu \mathrm{g}$ protein per lane was separated by electrophoresis by $10 \%$ SDS-PAGE and analyzed. Proteins were transferred onto a polyvinylidene difluoride membrane (Bio-Rad Laboratories, Inc.) and then blocked with 5\% dry milk in TBST (TBS with $0.1 \%$ Tween 20 ) for $1 \mathrm{~h}$ at room temperature, washed, and incubated overnight at $4^{\circ} \mathrm{C}$ with primary antibodies against JAK1 (1:1,000; cat. no. ab47435; Abcam, Cambridge, MA, USA), STAT3 (1:1,000; cat. no. ab68153; Abcam), phosphorylated (p)-STAT3 (1:800; cat. no. ab30647; Abcam), Bcl-2 (1:1,000; cat. no. 15071; Cell Signaling Technology, Inc., Danvers, MA, USA), c-myc (1:1,000; cat. no. 13987; Cell Signaling Technology, Inc.) and GAPDH (1:1,000; cat. no. 2118; Cell Signaling Technology, Inc.). The membranes were then washed 
Table I. Primers used in the reverse transcription quantitative polymerase chain reaction assay.

\begin{tabular}{ll} 
Genes & \multicolumn{1}{c}{ Sequence (5'-3') } \\
\hline hsa_circ_0044073F & ACAGGGGTGTTTGTGTGTGT \\
hsa_circ_0044073R & CTTCACGTTGCAGGTGTAGC \\
miR-107 F & ACACTCCAGCTGGGAGCAGCA \\
& UUGUACAGGG \\
miR-107 R & CTCAACTGGTGTCGTGGA \\
U6 F & CTCGCTTCGGCAGCACA \\
U6 R & AACGCTTCACGAATTTGCGT \\
JAK1 F & CACCAGGATGCGGATAAATAAT \\
JAK1 R & CAAAGTTTCCAAGGTAGCCAAG \\
STAT3 F & TGGTGTCTCCACTGGTCTATCTC \\
STAT3 R & CATCAATGAATCTAAAGTGCGG \\
Bcl-2 F & ATCGCCCTGTGGATGACTG \\
Bcl-2 R & AGACAGCCAGGAGAAATCAAAC \\
c-myc F & CATACATCCTGTCCGTCCA \\
c-myc R & CGCACAAGAGTTCCGTAGC \\
GAPDH F & TGTTCGTCATGGGTGTGAAC \\
GAPDH R & ATGGCATGGACTGTGGTCAT
\end{tabular}

F, forward; R, reverse; hsa, Homo sapiens; miR, microRNA; JAK1, janus kinase 1; STAT3, signal transducer and activator of transcription 3; Bcl-2, B-cell lymphoma 2; c-myc, v-myc avian myelocytomatosis viral oncogene homolog.

with TBST and then incubated with the secondary antibodies anti-rabbit and anti-mouse immunoglobulin $\mathrm{G}$ conjugated with horseradish peroxidase (HRP; 1:10,000; cat. no. 7074 and 7076; Cell Signaling Technology, Inc.). Finally, the immunoreactive bands were visualized by using an enhanced chemiluminescent substrate (ECL-Plus; GE Healthcare, Chicago, IL USA). Using ImageJ software (version 1.8.0; National Institutes of Health, Bethesda, MD, USA; http://imagej.nih.gove/ij/), densitometric analysis of bands was performed.

ELISA. The IL-1 $\beta$ (cat. no. CSB-E08053h), IL-6 (cat. no. CSB-E04638h) and TNF- $\alpha$ (cat. no. CSB-E04740h) ELISA assay kits were purchased from CUSABIO(Wuhan,China). After centrifugation at $1,000 \mathrm{xg}$ for $15 \mathrm{~min}$ at $4^{\circ} \mathrm{C}$, the concentrations in the supernatants were assessed according to the manufacturer's protocols. In brief, the samples were added to wells in duplicate and then incubated at room temperature for $2 \mathrm{~h}$. The liquid was removed, followed by the addition of biotin-antibody and then the plates were incubated at room temperature for $1 \mathrm{~h}$. Following removal of the liquid and washing, HRP-avidin was added and incubated at room temperature for $1 \mathrm{~h}$. Following additional wash steps, visualization was achieved by adding the TMB substrate. The absorbance was detected at $450 \mathrm{~nm}$ with a microplate reader (Thermo Fisher Scientific, Inc.). The experiments were performed in triplicate.

Cell cycle analysis by flow cytometry. The cell cycle distribution was analyzed as described previously (29). Following treatment with LPS for $24 \mathrm{~h}$, the cells were collected and washed with PBS and then stained with $50 \mathrm{mg} / \mathrm{ml}$ propidium iodide (Nanjing KeyGen Biotech Co., Ltd., Nanjing, China) for $30 \mathrm{~min}$ at $4^{\circ} \mathrm{C}$. The data were collected with a FACSCalibur flow cytometer (Becton, Dickinson and Company, Franklin Lakes, NJ, USA) for cell-cycle distribution analysis using Cell Quest Software (version 5.1; Becton, Dickinson and Company).

Matrigel invasion assay. The cells were collected and suspended in medium without serum. Transwell inserts (24-well insert; pore size, $8 \mathrm{~mm}$; Becton, Dickinson and Company) were inserted in the chamber and incubated at $37^{\circ} \mathrm{C}$ overnight with serum-free RPMI-1640 with matrigel (0.6 mg/ml; Becton, Dickinson and Company). Subsequently, $4 \times 10^{4}$ cells in $0.2 \mathrm{ml}$ serum-free medium were seeded in the top chambers of the transwell inserts, and 10\% FBS was used as an attractant. The cells were incubated with $1 \mathrm{mg} / \mathrm{ml} \mathrm{LPS}$ or control treatment for $48 \mathrm{~h}$ at $37^{\circ} \mathrm{C}$. The cells that did not invade through the pores were removed, and the filters were stained at room temperature with hematoxylin for $5 \mathrm{~min}$, and with eosin for $1 \mathrm{~min}$ (cat. no. C0105; Beyotime Institute of Biotechnology, Haimen, China). Samples were visualized under a light microscope (magnification, x200) and counting.

RNA pull-down assay. The RNA pull-down assay was performed as previously described (30). In brief, either the pAd-Track-cmv-circRNA-0044073 or the control vectors were transfected into 293 cells (ATCC). Subsequently, $100 \mu \mathrm{g}$ total RNA was extracted with TRIzol ${ }^{\circledR}$ (Invitrogen; Thermo Fisher Scientific, Inc.) from the cells and incubated with $500 \mu \mathrm{g}$ streptavidin magnetic beads (cat. no. S1421S; New England BioLabs, Inc., Ipswich, MA, USA), which were previously incubated for $2 \mathrm{~h}$ at $37^{\circ} \mathrm{C}$ with either 200 pmol biotin-miR-107 or 200 pmol biotin-miR-107-mut (Shanghai GenePharma Co., Ltd., Shanghai, China). Following RNA elution, RT-qPCR was performed to detect circRNA-0044073 as aforementioned.

DNA construct and luciferase reporter assay. As described previously (18), the psiCHECKTM-2 firefly luciferase reporter plasmids (Promega Corporation, Madison, WI, USA) were recombined with the potential miR-107 binding site sequences of JAK1 or its mutated sequence. 293 cells were seeded into a 12 -well plate $\left(3 \times 10^{5}\right.$ cells/well) and transfected with $200 \mathrm{ng}$ recombinant luciferase reporter plasmid using Lipofectamine ${ }^{\circledR}$ 2000 (Invitrogen; Thermo Fisher Scientific, Inc.). Following transfection with $10 \mathrm{nM}$ miR-107 mimics (5'-AGCAGCAUU GUACAGGGCUAUCA-3') or NC miRNA (5'UUCUCCGAA CGUGUCACGUTT-3') for $24 \mathrm{~h}$, the cells were collected, and luciferase activity was determined using the Dual-Luciferase Reporter Gene Assay kit (Beyotime Institute of Biotechnology). The relative luciferase activity was obtained by normalizing the firefly luciferase activity to the internal control Renilla luciferase activity.

Statistical analysis. Data in the present study are presented as the mean \pm standard deviation of three independent tests. Using GraphPad Prism (version 5.0; GraphPad Software, Inc., La Jolla, CA, USA), two-tailed Student's t-tests were performed to compare data between two groups, whereas, one-way analysis of variance followed by Tukey's post hoc test was performed to compare data among three or more groups. 
A

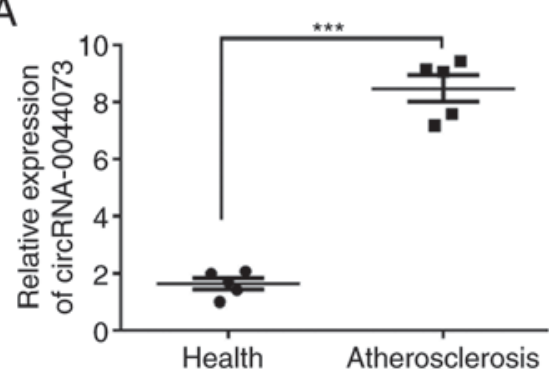

B

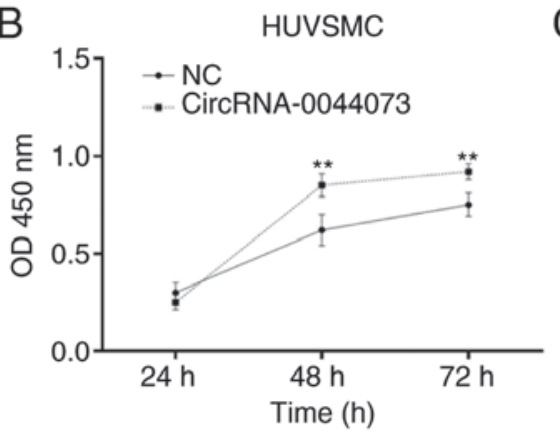

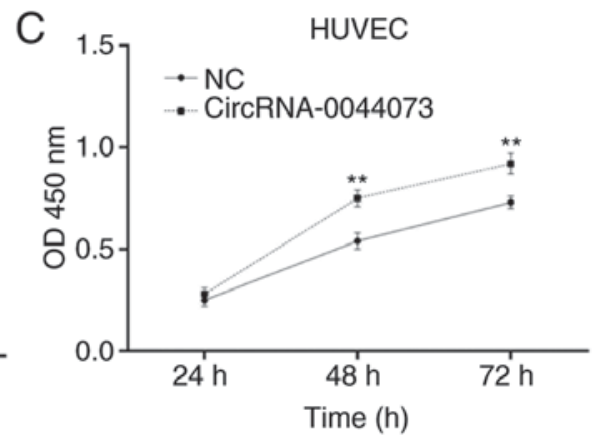

Figure 1. Dysregulated expression levels of circRNA-0044073 in blood cells of patients with atherosclerosis and its role in cell proliferation. (A) Expression levels of circRNA-0044073 from blood cells from 5 randomly selected patients with atherosclerosis and 5 healthy donor livers were detected. (B) CircRNA-0044073 significantly promoted the proliferation of HUVSMCs. (C) CircRNA-0044073 significantly enhanced the proliferation of $\mathrm{HUVECs}$. ${ }^{* *}<0.01$ and ${ }^{* * *} \mathrm{P}<0.001$ vs. control. circRNA, circular RNA; HUVSMCs, human vascular smooth muscle cells; HUVECs, human vascular endothelial cells; NC, negative control; Health, health donor samples.

\section{Results}

Levels of circRNA-0044073 are upregulated in blood cells of patients with atherosclerosis and are associated with the proliferation of HUVSMCs and HUVECs. The present study identified that the expression of circRNA-0044073 in blood cells was significantly increased in patients with atherosclerosis compared with that of healthy controls $(\mathrm{P}<0.001$; Fig. 1A). Therefore, we hypothesized that circRNA-0044073 serves a key role in atherosclerosis. As the abnormal proliferation of ECs, SMCs and macrophages contribute to the progression of atherosclerosis, the in vitro protocols of the present study included HUVECs and HUVSMCs to determine the effect of circRNA-0044073 on cell growth. It was observed that the overexpression of circRNA-0044073 significantly increased the proliferation of HUVECs and HUVSMCs, compared with control groups, at 48 and $72 \mathrm{~h}(\mathrm{P}<0.01$; Fig. $1 \mathrm{~B}$ and $\mathrm{C})$. These results suggest a potential association between the upregulation of circRNA-0044073 and proliferation of atherosclerosis-associated cells.

Levels of miR-107 are downregulated in blood cells of patients with atherosclerosis and is targeted by circRNA-0044073. A previous study has suggested the involvement of miR-107 in the development of atherosclerosis (26). Therefore, the levels of miR-107 were detected and compared between patients with atherosclerosis and healthy individuals. Notably, the expression of miR-107 was significantly decreased in the blood cells of patients with atherosclerosis compared with the controls $(\mathrm{P}<0.05$; Fig. 2A). In addition, miR-107 mimics significantly inhibited the proliferation of HUVECs and HUVSMCs, compared with the control groups, at 48 and $72 \mathrm{~h}$, which was in contrast to the observed effects of circRNA-0044073 ( $\mathrm{P}<0.01$; Fig. 2B and C). The association between circRNA-0044073 and miR-107 was then investigated; the data suggested that the overexpression of circRNA-0044073 significantly downregulated the levels of miR-107 in HUVECs and HUVSMCs $(\mathrm{P}<0.01$; Fig. 2D and E). Furthermore, RNA pull down assay demonstrated that miR-107 was a target of circRNA-0044073 ( $\mathrm{P}<0.01$; Fig. 2D-F). These results indicate that circRNA-0044073 counteracts the effect of miR-107 via a sponge mechanism.
CircRNA-0044073 increases the proliferation and invasion of cells by regulating miR-107. The regulatory mechanism of circRNA-0044073 on the invasive activity of HUVSMCs and HUVECs was then explored. As an endotoxin, LPS is a potentially important stimulator and atherosclerosis risk factor. Therefore, an in vitro atherosclerosis model was established by treating cells with LPS. Compared with the control groups, treatment with LPS significantly increased the number of invasive HUVSMCs and inhibited the proliferation of HUVECs, respectively ( $\mathrm{P}<0.01$; Fig. 3A and B). Additionally, LPS treatment significantly increased the levels of circRNA-0044073 in HUVSMCs but downregulated the levels of circRNA-0044073 in HUVECs ( $\mathrm{P}<0.001$; Fig. 3C). It was additionally identified that in HUVSMCs, the combined use of LPS and circRNA-0044073 significantly increased the proliferation of cells, compared with a single treatment of LPS or circRNA-0044073 overexpression vector $(\mathrm{P}<0.01$; Fig. 3D and E). By contrast, miR-107 mimics significantly alleviated the effect of LPS + circRNA-0044073 treatment on the promotion the proliferation of cells $(\mathrm{P}<0.01$; Fig. 3D-E). Although the invasive activity of HUVECs was suppressed by LPS, circRNA-0044073 transfection partially reversed the effect of LPS and induced the invasion of HUVECs. Similar to the effect in HUVSMCs, miR-107 impeded the role of circRNA-0044073 in promoting cell proliferation. The effect of circRNA-0044073 on the cell cycle distribution was also examined. Despite the observation that LPS treatment affected the percentages of G2/M and S phase cells, with the addition of circRNA-0044073 transfection, the percentage of cells in G2/M phase was additionally increased, and was partially impaired by using miR-107 mimics (Fig. 4). These results support the hypothesis that circRNA-0044073 facilitates the proliferation and invasion of cells by inhibiting miR-107 in the development of atherosclerosis.

CircRNA-0044073 activates the JAK/STAT signaling pathway of HUVSMCs and HUVECs. JAK1 is a critical contributor to the pathogenesis of a number of inflammatory diseases and has been identified as a potential therapeutic target $(31,32)$. The expression levels of JAK1 and STAT3 were significantly increased in blood cells in patients with atherosclerosis, in addition to an increase in Bcl-2 and c-myc levels, compared with those in the healthy controls $(\mathrm{P}<0.001$; Fig. 5A-D). Notably, in 
A

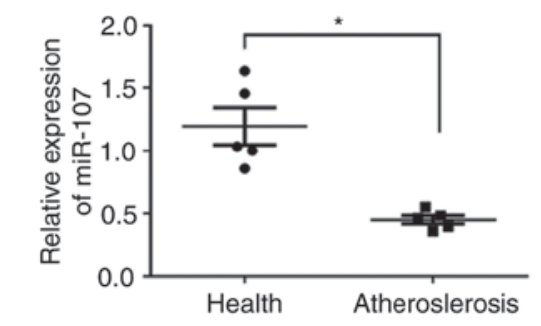

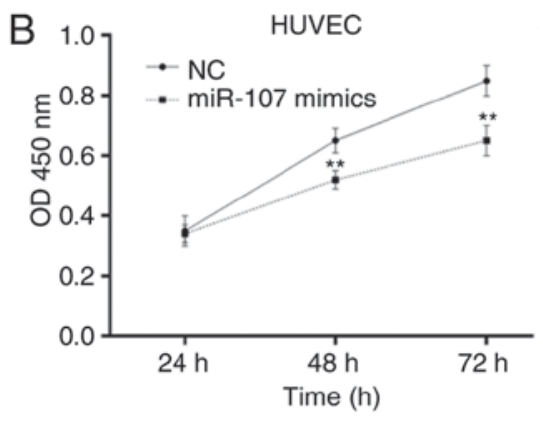

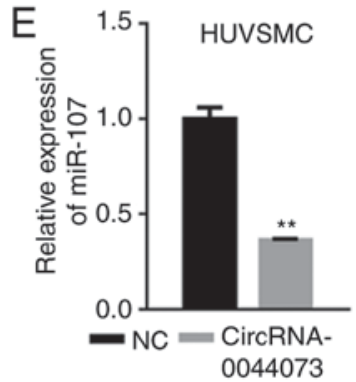

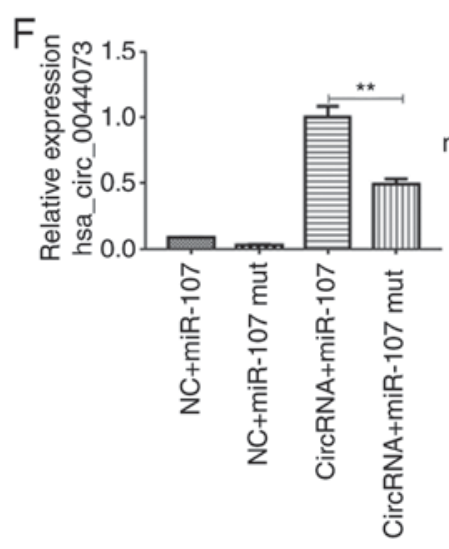

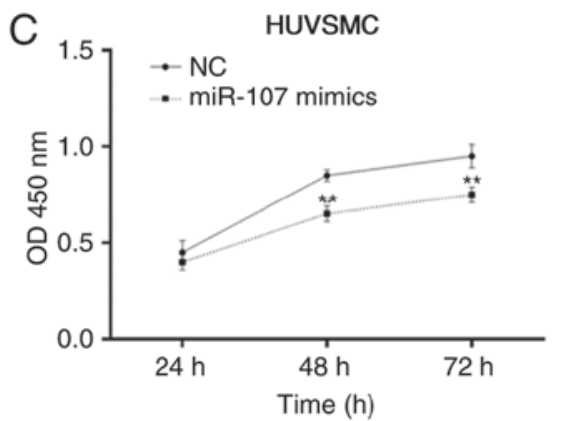

miR-107 WT 3'-ACAUGUUACGACGA-5' || || |ा|

CircRNA 5'-CATACA CTGC TGTC-3' miR-107 MUT 3 '-ACTGTCGTAA CAGA-5'

Figure 2. Effect of miR-107 in atherosclerosis and negative associations with circRNA-0044073. (A) Expression levels of miR-107 from blood cells from 5 randomly selected patients with atherosclerosis and 5 healthy-donor livers were detected. (B) miR-107 significantly inhibited the proliferation of HUVSMCs. (C) miR-107 significantly inhibited the proliferation of HUVECs. (D) CircRNA-0044073 significantly downregulated the level of miR-107 in HUVSMCs. (E) CircRNA-0044073 significantly decreased the level of miR-107 in HUVECs. (F) Targeting of miR-107 by circRNA-0044073 was determined by RNA pull-down assay. ${ }^{*} \mathrm{P}<0.05,{ }^{* *} \mathrm{P}<0.01$ and ${ }^{* * *} \mathrm{P}<0.001$ vs. control. miR, microRNA; circRNA, circular RNA; HUVSMCs, human vascular smooth muscle cells; HUVECs, human vascular endothelial cells; NC, negative control; Health, health donor samples.

the LPS treatment group, the Bcl-2 and c-myc levels were markedly increased in HUVSMCs and decreased in HUVECs, which is consistent with the distinct effect of LPS on the proliferation of these two types of cells $(33,34)$. However, the overexpression of miR-107 significantly inhibited the levels of JAK1 in HUVSMCs and HUVECs (Fig. 5E-F). The luciferase reporter assay additionally confirmed that JAK1 was a target of miR-107 (Fig. 5G). In contrast to the inhibitory role of miR-107 on JAK1 expression, overexpression of circRNA-0044073 activated the JAK/STAT signaling pathway, along with upregulation of Bcl-2, c-myc, IL-1 $\beta$, IL-6 and TNF- $\alpha$ levels, compared with those in control group, which is in accordance with the in vivo data concerning the changes in expression of JAK1, p-STAT3, Bcl-2 and c-myc (Fig. 5H-N).

\section{Discussion}

As the primary cause of myocardial infarction, heart failure, myocardial ischemia and stroke, atherosclerosis accounts for high mortality and morbidity rates worldwide (35). Although a number of treatments have been developed and widely applied for atherosclerosis, certain subgroups of patients remain at high risk for the development and progression of atherosclerosis, creating an urgent requirement to explore novel therapeutic targets or/and more effective treatments for atherosclerosis. A previous study demonstrated that circRNAs are crucial contributors to the pathogenesis of atherosclerosis (36). Therefore, increasing interest in the treatment of atherosclerosis is being focused on circRNAs at present. In the present study, to the best of our knowledge, it was observed for the first time that circRNA-0044073 levels were negatively associated with atherosclerosis and that cirRNA-0044073 may directly target miR-107 and thereby lead to the upregulation of JAK1/STAT3 signaling.

As a chronic inflammatory disease, atherosclerosis constitutes a dysregulation of a variety of cytokines. A previous study demonstrated that the JAK/STAT signaling pathway promotes vascular cell inflammation, proliferation, migration and adhesion (37). JAK1 is expressed in all cell types, including ECs and SMCs $(38,39)$. Among the STAT proteins, STAT3 is an activator of systemic inflammatory genes and is present in the inflammatory regions of human atherosclerotic lesions in an activated form. Previous data demonstrated an important role for the JAK/STAT pathway in oxidized 1-palmitoyl-2-arachidonoyl-sn-glycero-3-phosphocholine-induced IL-8 transcription in vitro and in atherosclerosis in vivo (1). It has also been indicated that suppressors of cytokine signaling modulate the JAK/STAT-dependent responses in vascular cells, which are associated with atherosclerotic plaque development (40). In the present study, it was observed that the expression levels of JAK1 and STAT3 were significantly increased in the blood cells from patients with atherosclerosis, which was consistent with previous studies $(18,41)$. Bcl-2 proteins represent the major regulators of extrinsic and intrinsic apoptosis signaling pathways and target apoptosis of vascular cells in atherosclerotic lesions. The decrease in c-myc oncogene levels alleviates the proliferation in rat VSMCs and induces apoptosis (42). Therefore, Bcl-2 and c-myc serve important roles in the deregulation of cell apoptosis and 

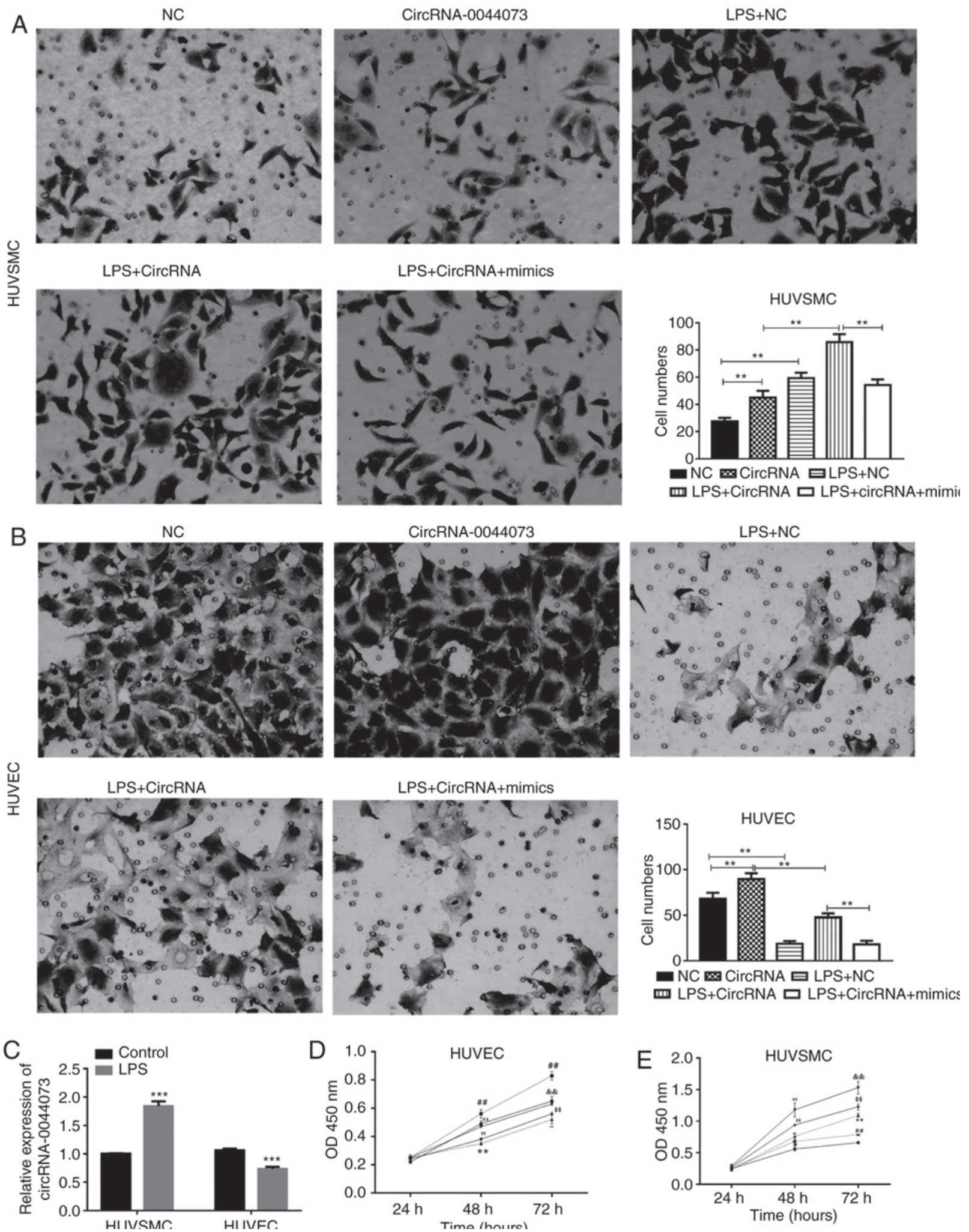

Figure 3. Effect of circRNA-0044073 on cell proliferation and invasion of HUVSMCs and HUVECs. (A) LPS induced the proliferation of HUVSMCs. (B) LPS facilitated the proliferation of HUVECs. (C) LPS affected the level of circRNA-0044073 in the HUVSMCs and HUVECs. (D) The representative images and histograms indicate the numbers of the migrated HUVSMCs (magnification, 100x). (E) The representative images and histogram indicate the numbers of the migrated HUVECs (magnification, 100x). ${ }^{* *} \mathrm{P}<0.01$ and ${ }^{* * *} \mathrm{P}<0.001$ vs. control; \&\& $\mathrm{P}<0.01$ LPS $+\operatorname{circRNA}$ vs. LPS $+\mathrm{NC}$; ${ }^{\# /} \mathrm{P}<0.01 \mathrm{CircRNA}$ vs. NC; P $<0.01$ LPS+circRNA+mimics vs. LPS+circRNA. circRNA, circular RNA; HUVSMCs, human vascular smooth muscle cells; HUVECs, human vascular endothelial cells; NC, negative control; LPS, lipopolysaccharide.

proliferation, contributing to the pathogenesis of atherosclerosis (43). The data presented in the present study indicated that the expression levels of $\mathrm{Bcl}-2$ and c-myc were significantly increased in the blood cells from patients with atherosclerosis compared with the healthy controls. Consistent with these results, a previous study revealed that c-myc was overexpressed 

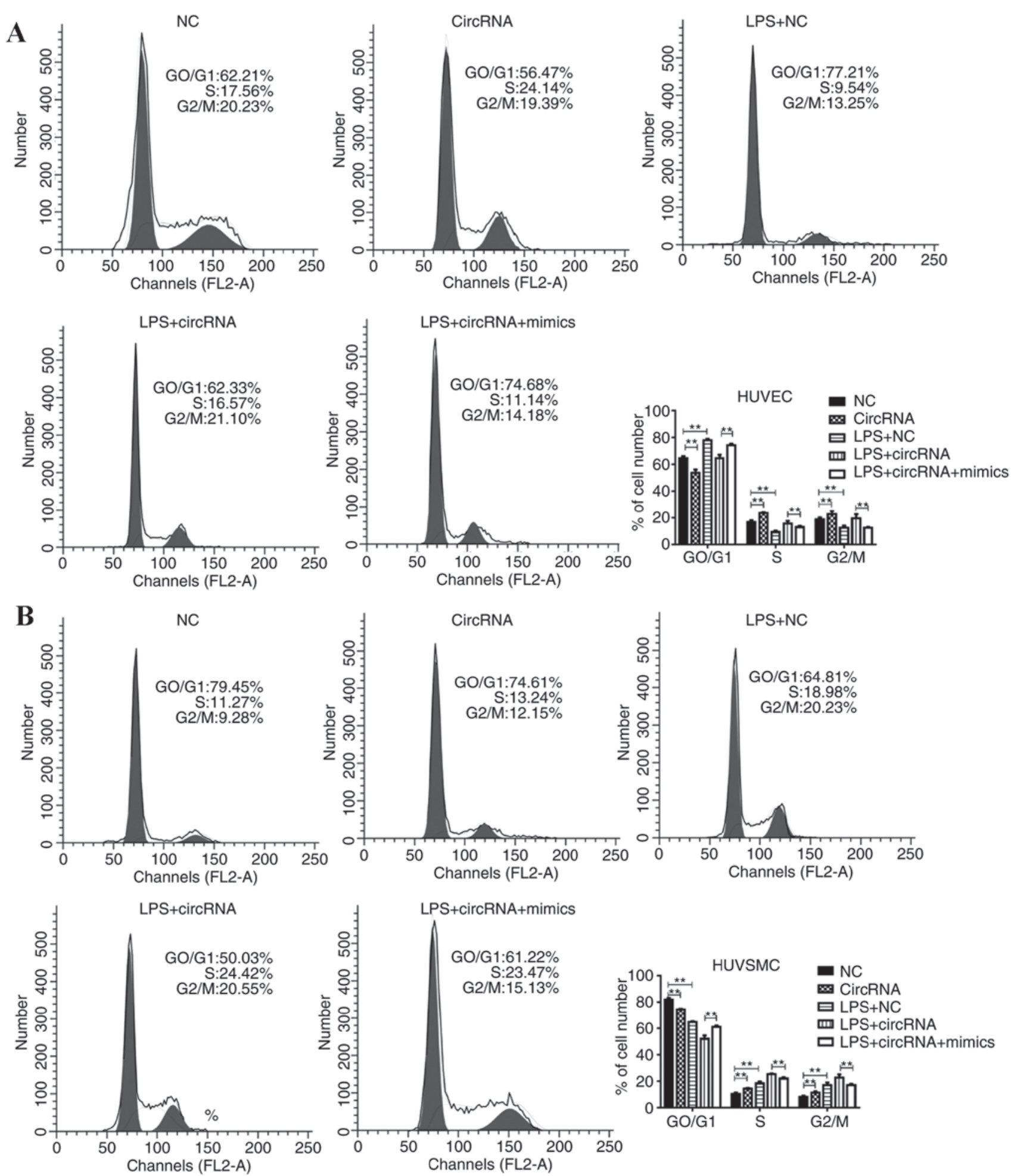

Figure 4. Effect of circRNA-0044073 on cell cycle. Cell cycle phases of (A) HUVECs and (B) HUVSMCs. *"P<0.01 vs. NC. circRNA, circular RNA; HUVSMCs, human vascular smooth muscle cells; HUVECs, human vascular endothelial cells; NC, negative control.

in plaque SMCs (44). However, a small number of studies contradict the Bcl-2 data from the present study $(45,46)$. This may be due to differences in the test samples and the stage of atherosclerosis investigated.

HUVECs have been used as in vitro models for the study of atherosclerosis (47). As an endotoxin, LPS is a potentially important stimulator and risk factor for atherosclerosis (48). LPS has been implicated in endothelial injury and has induced apoptosis and proliferation in endothelial and VSMCs, respectively $(34,35,49)$. Consistently, the present study observed that LPS exposure resulted in the apoptosis of HUVECs and the proliferation of HUVSMCs, and the increased expression of circRNA-0044073 in the cells. With the proliferative effect of circRNA-0044073, the apoptosis of HUVECs by LPS was significantly attenuated, and the proliferation of HUVSMCs was significantly improved. Furthermore, it was also observed 

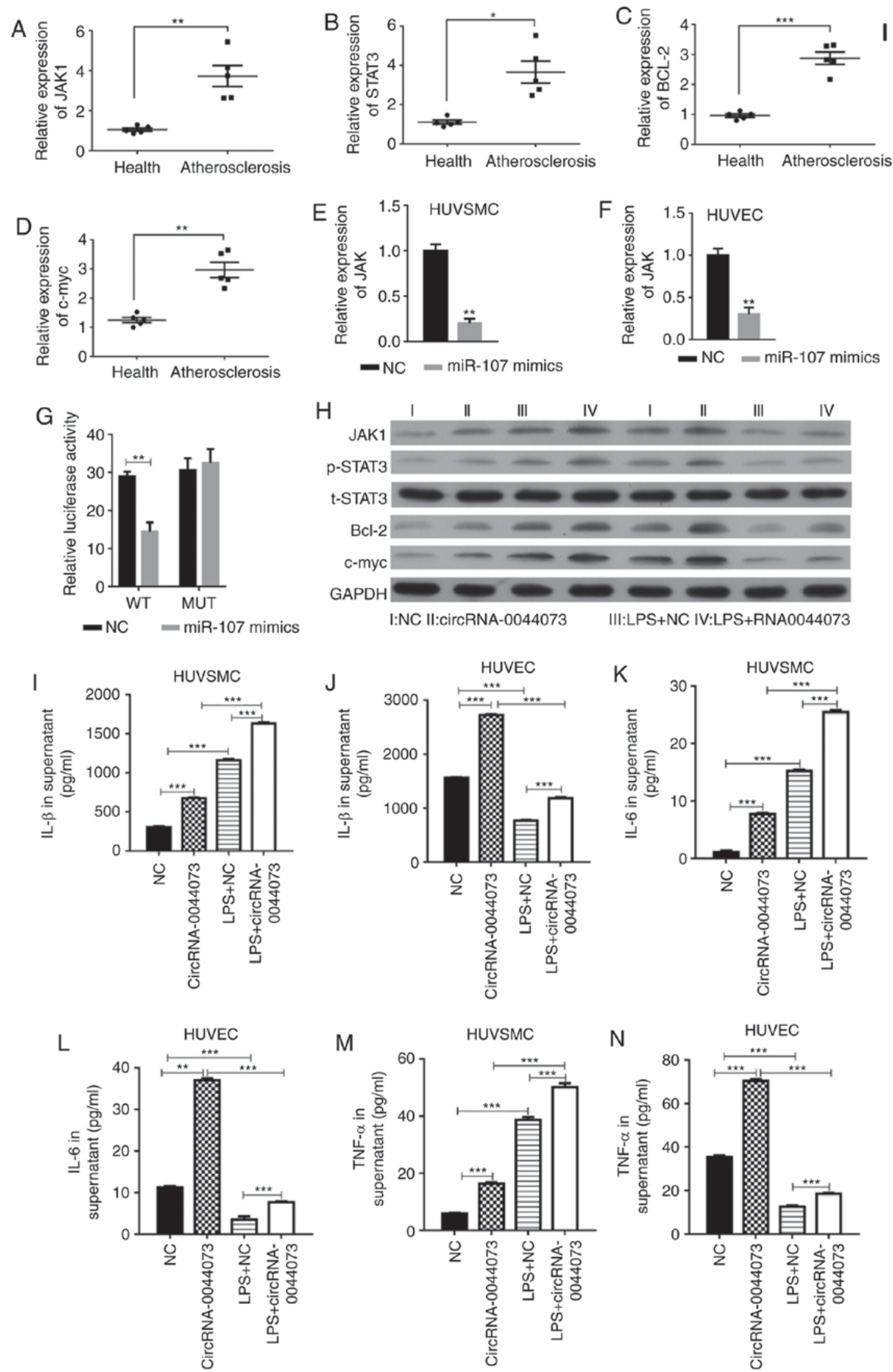

Figure 5. Effects of circRNA-0044073 on the JAK/STAT pathway and inflammation in atherosclerosis. Expression levels of (A) JAK1, (B) STAT3, (C) Bcl-2 and (D) c-myc from blood cells from 5 randomly selected patients with atherosclerosis and 5 healthy donor livers were detected. miR-107 significantly downregulated the expression levels of JAK1 in (E) HUVSMCs and (F) HUVECs. (G) The role of JAK1 as a target of miR-107 was identified by using a luciferase reporter assay. (H) JAK1, t-STAT3, p-STAT3, Bcl-2, c-myc levels were analyzed by western blot analysis. Expression levels of (I) IL-1 $\beta$ in HUVSMCs, (J) IL-1 $\beta$ in HUVECs, (K) IL-6 in HUVSMCs, (L) IL-6 in HUVECs, (M) TNF- $\alpha$ in HUVSMCs and (N) TNF- $\alpha$ in HUVECs were analyzed by ELISA. "P<0.05, ${ }^{* *} \mathrm{P}<0.01$ and ${ }^{* * *} \mathrm{P}<0.001$ vs. NC. circRNA, circular RNA; HUVSMCs, human vascular smooth muscle cells; HUVECs, human vascular endothelial cells; NC, negative control; Il, interleukin; TNF- $\alpha$, tumor necrosis factor $\alpha$; JAK1, janus kinase 1; STAT3, signal transducer and activator of transcription 3; Bcl-2, B-cell lymphoma 2; c-myc, v-myc avian myelocytomatosis viral oncogene homolog; t-STAT3, total STAT3; p, phosphorylated; Health, health donor samples. 
that the invasion levels of HUVECs and HUVSMCs were promoted on treatment with LPS. Therefore, we hypothesized that circRNA-0044073 is associated with the initiation and development of the disease. However, additional studies are required to validate this.

miR-107 is associated with inflammation (23). Previously, it was demonstrated that the expression of miR-107 is decreased in macrophages following LPS stimulation $(23,50)$. A consistent change in miR-107 expression in the blood cells from patients with atherosclerosis was observed in the present study. However, it was also demonstrated that the miR-107 expression was upregulated in HUVECs and HUVSMCs following LPS treatment. This variation may be due to the different cells examined. Based on the actual pro-inflammation function of miR-107, the decrease in miR-107 expression may be a result of the regulative feedback (23). Consistent with a previous study, the present study confirmed that miR-107 directly targeted JAK1 (51). Additionally, it was demonstrated that miR-107 is also a direct target of circRNA-0044073. Furthermore, it was observed that in HUVSMCs and HUVECs, circRNA-0044073 overexpression decreased miR-107 levels and resultantly increased the levels of JAK1 and p-STAT3, and the levels of downstream proteins including Bcl-2 and c-myc. As JAK1/STAT3 serve critical roles in inflammation, we hypothesized that cirRNA-0044073 may be associated with inflammation, which is a key contributor to atherosclerosis. In the present study, it was observed that circRNA-0044073 significantly induced the levels of IL-1 $\beta$, IL- 6 and TNF- $\alpha$. These pro-inflammatory cytokines are pro-atherogenic. However, the underlying mechanisms by which circRNA-0044073 regulates these inflammatory cytokines requires additional investigation.

Taken together, the data from the present study suggests that circRNA-0044073 is a potential therapeutic target for the treatment of atherosclerosis, as it directly targets miR-107 and resultantly increases STAT3 activation, and upregulates the expression of the downstream proteins that contribute to atherosclerosis.

\section{Acknowledgements}

Not applicable.

\section{Funding}

The present study was supported by Key Research and Development Plan of Shandong Province (grant no. 2017GSF218012) and Shandong Provincial Natural Science Foundation (grant no. ZR2015HM052).

\section{Authors' contributions}

LS, YH and WWu designed the study. LS, YH, JL, SY, WWa and YW performed the experiments. LS, YX and WWa performed the data analysis and drafted the manuscript. All authors read and approved the final manuscript.

\section{Availability of data and materials}

The datasets used and/or analyzed during the current study are available from the corresponding author on reasonable request.

\section{Ethics approval and consent to participate}

Informed consent was obtained from all patients and ethical approval was granted by The Ethics Committee of the Qi-Lu Hospital of Shandong University.

\section{Patient consent for publication}

Informed consent was obtained from all patients.

\section{Competing interests}

The authors declare that they have no competing interests.

\section{References}

1. Gharavi NM, Alva JA, Mouillesseaux KP, Lai C, Yeh M, Yeung W, Johnson J, Szeto WL, Hong L, Fishbein M, et al: Role of the Jak/STAT pathway in the regulation of interleukin-8 transcription by oxidized phospholipids in vitro and in atherosclerosis in vivo. J Biol Chem 282: 31460-31468, 2007.

2. Schober A, Nazari-Jahantigh M, Wei Y, Bidzhekov K, Gremse F, Grommes J, Megens RT, Heyll K, Noels H, Hristov M, et al: MicroRNA-126-5p promotes endothelial proliferation and limits atherosclerosis by suppressing Dlk1. Nat Med 20: 368-376, 2014.

3. Gorenne I, Kumar S, Gray K, Figg N, Yu H, Mercer J and Bennett M: Vascular smooth muscle cell sirtuin 1 protects against DNA damage and inhibits atherosclerosis. Circulation 127: 386-396, 2013.

4. Zheng Y, Li Y, Liu G, Qi X and Cao X: MicroRNA-24 inhibits the proliferation and migration of endothelial cells in patients with atherosclerosis by targeting importin- $\alpha 3$ and regulating inflammatory responses. Exp Ther Med 15: 338-344, 2018.

5. Baumer Y,McCurdy S, Alcala M, Mehta N, Lee BH, Ginsberg MH and Boisvert WA: CD98 regulates vascular smooth muscle cell proliferation in atherosclerosis. Atherosclerosis 256: 105-114, 2017.

6. Luc G, Bard JM, Juhan-Vague I, Ferrieres J, Evans A, Amouyel P, Arveiler D, Fruchart JC, Ducimetiere P and PRIME Study Group: C-reactive protein, interleukin-6, and fibrinogen as predictors of coronary heart disease: The PRIME study. Arterioscler Thromb Vasc Biol 23: 1255-1261, 2003.

7. Brunetti ND, Salvemini G, Cuculo A, Ruggiero A, De Gennaro L, Gaglione A and Di Biase M: Coronary artery ectasia is related to coronary slow flow and inflammatory activation. Atherosclerosis 233: 636-640, 2014.

8. Zernecke A and Weber C: Chemokines in the vascular inflammatory response of atherosclerosis. Cardiovasc Res 86: 192-201, 2010.

9. Barton M: Prevention and endothelial therapy of coronary artery disease. Curr Opin Pharmacol 13: 226-241, 2013.

10. Holdt LM, Stahringer A, Sass K, Pichler G, Kulak NA, Wilfert W, Kohlmaier A, Herbst A, Northoff BH, Nicolaou A, et al: Circular non-coding RNA ANRIL modulates ribosomal RNA maturation and atherosclerosis in humans. Nat Commun 7: 12429, 2016.

11. Bayoumi AS, Aonuma T, Teoh JP, Tang YL and Kim IM: Circular noncoding RNAs as potential therapies and circulating biomarkers for cardiovascular diseases. Acta Pharmacol Sin 39: 1100-1109, 2018

12. Li CY, Ma L and Yu B: Circular RNA hsa_circ_0003575 regulates oxLDL induced vascular endothelial cells proliferation and angiogenesis. Biomed Pharmacother 95: 1514-1519, 2017.

13. Zheng C, Niu H, Li M, Zhang H, Yang Z, Tian L, Wu Z, Li D and Chen X: Cyclic RNA has-circ-000595 regulates apoptosis of aortic smooth muscle cells. Mol Med Rep 12: 6656-6662, 2015.

14. Diniz GP and Wang DZ: Regulation of skeletal muscle by microRNAs. Compr Physiol 6: 1279-1294, 2016.

15. Lu C, Xie Z and Peng Q: MiRNA-107 enhances chemosensitivity to paclitaxel by targeting antiapoptotic factor Bcl-w in non small cell lung cancer. Am J Cancer Res 7: 1863-1873, 2017.

16. Chen Z, Zhao L, Zhao F, Yang G and Wang J: MicroRNA-26b regulates cancer proliferation migration and cell cycle transition by suppressing TRAF5 in esophageal squamous cell carcinoma. Am J Transl Res 8: 1957-1970, 2016. 
17. Undi RB, Kandi R and Gutti RK: MicroRNAs as haematopoiesis regulators. Adv Hematol 2013: 695754, 2013.

18. Wang Y, Han Z, Fan Y, Zhang J, Chen K, Gao L, Zeng H, Cao J and Wang C: MicroRNA-9 Inhibits NLRP3 inflammasome activation in human atherosclerosis inflammation cell models through the JAK1/STAT signaling pathway. Cell Physiol Biochem 41: 1555-1571, 2017.

19. Jiang ZP and Zhou TB: Role of miR-107 and its signaling pathways in diseases. J Recept Signal Transduct Res 34: 338-341, 2014.

20. Liu F, Liu S, Ai F, Zhang D, Xiao Z, Nie X and Fu Y: miR-107 promotes proliferation and inhibits apoptosis of colon cancer cells by targeting prostate apoptosis response-4 (Par4). Oncol Res 25: 967-974, 2017.

21. Nelson PT and Wang WX: MiR-107 is reduced in Alzheimer's disease brain neocortex: Validation study. J Alzheimers Dis 21: 75-79, 2010.

22. Su SG, Yang M, Zhang MF, Peng QZ, Li MY, Liu LP and Bao SY: miR-107-mediated decrease of HMGCS2 indicates poor outcomes and promotes cell migration in hepatocellular carcinoma. Int J Biochem Cell Biol 91: 53-59, 2017.

23. Foley NH and O'Neill LA: miR-107: A Toll-like receptor-regulated miRNA dysregulated in obesity and type II diabetes. J Leukoc Biol 92: 521-527, 2012.

24. Li F, Liu B, Gao Y, Liu Y, Xu Y, Tong W and Zhang A: Upregulation of MicroRNA-107 induces proliferation in human gastric cancer cells by targeting the transcription factor FOXO1. FEBS Lett 588: 538-544, 2014

25. Norata GD, Sala F, Catapano AL and Fernandez-Hernando C: MicroRNAs and lipoproteins: A connection beyond atherosclerosis? Atherosclerosis 227: 209-215, 2013.

26. Daimiel-Ruiz L, Klett-Mingo $M$, Konstantinidou V Micó V, Aranda JF, García B, Martínez-Botas J, Dávalos A, Fernández-Hernando C and Ordovás JM: Dietary lipids modulate the expression of miR-107, an miRNA that regulates the circadian system. Mol Nutr Food Res 59: 552-565, 2015.

27. Hansen TB, Jensen TI, Clausen BH, Bramsen JB, Finsen B, Damgaard CK and Kjems J: Natural RNA circles function as efficient microRNA sponges. Nature 495: 384-388, 2013.

28. Livak KJ and Schmittgen TD: Analysis of relative gene expression data using real-time quantitative PCR and the 2(-Delta Delta C(T)) method. Methods 25: 402-408, 2001.

29. Kong X, Moran MS, Zhao Y and Yang Q: Inhibition of metadherin sensitizes breast cancer cells to AZD6244. Cancer Biol Ther 13: 43-49, 2012.

30. Tang CM, Zhang M, Huang L, Hu ZQ, Zhu JN, Xiao Z, Zhang Z, Lin QX, Zheng XL, Yang M, et al: CircRNA_000203 enhances the expression of fibrosis-associated genes by derepressing targets of miR-26b-5p, Colla 2 and CTGF, in cardiac fibroblasts Sci Rep 7: 40342, 2017.

31. O'Shea JJ and Plenge R: JAK and STAT signaling molecules in immunoregulation and immune-mediated disease. Immunity 36 542-550, 2012.

32. Maschalidi S, Sepulveda FE, Garrigue A, Fischer A and de Saint Basile G: Therapeutic effect of JAK1/2 blockade on the manifestations of hemophagocytic lymphohistiocytosis in mice. Blood 128: 60-71, 2016

33. Choi KB, Wong F, Harlan JM, Chaudhary PM, Hood L and Karsan A: Lipopolysaccharide mediates endothelial apoptosis by a FADD-dependent pathway. J Biol Chem 273: 20185-20188, 1998.
34. Jiang D, Yang Y and Li D: Lipopolysaccharide induced vascular smooth muscle cells proliferation: A new potential therapeutic target for proliferative vascular diseases. Cell Prolif 50: e12332, 2017.

35. Liu M, Tao G, Liu Q, Liu K and Yang X: MicroRNA let-7g alleviates atherosclerosis via the targeting of LOX-1 in vitro and in vivo. Int J Mol Med 40: 57-64, 2017.

36. Burd CE, Jeck WR, Liu Y, Sanoff HK, Wang Z and Sharpless NE: Expression of linear and novel circular forms of an INK4/ARF-associated non-coding RNA correlates with atherosclerosis risk. PLoS Genet 6: e1001233, 2010.

37. Wang R, Zhang Y, Xu L, Lin Y, Yang X, Bai L, Chen Y, Zhao S, Fan J, Cheng X and Liu E: Protein inhibitor of activated STAT3 suppresses oxidized LDL-induced cell responses during atherosclerosis in apolipoprotein E-deficient mice. Sci Rep 6: 36790, 2016.

38. Rane SG and Reddy EP: Janus kinases: Components of multiple signaling pathways. Oncogene 19: 5662-5679, 2000

39. Verma A, Kambhampati S, Parmar S and Platanias LC: Jak family of kinases in cancer. Cancer Metastasis Rev 22: 423-434, 2003.

40. Ortiz-Muñoz G, Martin-Ventura JL, Hernandez-Vargas $P$, Mallavia B, Lopez-Parra V, Lopez-Franco O, Muñoz-Garcia B, Fernandez-Vizarra P, Ortega L, Egido J and Gomez-Guerrero C: Suppressors of cytokine signaling modulate JAK/STAT-mediated cell responses during atherosclerosis. Arterioscler Thromb Vasc Biol 29: 525-531, 2009.

41. Hiltunen MO, Tuomisto TT, Niemi M, Bräsen JH, Rissanen TT, Törönen P, Vajanto I and Ylä-Herttuala S: Changes in gene expression in atherosclerotic plaques analyzed using DNA array. Atherosclerosis 165: 23-32, 2002.

42. Bennett MR, Evan GI and Newby AC: Deregulated expression of the c-myc oncogene abolishes inhibition of proliferation of rat vascular smooth muscle cells by serum reduction, interferon-gamma, heparin, and cyclic nucleotide analogues and induces apoptosis. Circ Res 74: 525-536, 1994.

43. Kutuk $\mathrm{O}$ and Basaga $\mathrm{H}$ : $\mathrm{Bcl}-2$ protein family: Implications in vascular apoptosis and atherosclerosis. Apoptosis 11: 1661-1675, 2006.

44. Marin ML, Gordon RE, Veith FJ, Tulchin N and Panetta TF: Distribution of c-myc oncoprotein in healthy and atherosclerotic human carotid arteries. J Vasc Surg 18: 170-177, 1993.

45. Kockx MM, De Meyer GR, Muhring J, Jacob W, Bult H and Herman AG: Apoptosis and related proteins in different stages of human atherosclerotic plaques. Circulation 97: 2307-2315, 1998.

46. Saxena A, McMeekin JD and Thomson DJ: Expression of Bcl-x, Bcl-2, Bax, and Bak in endarterectomy and atherectomy specimens. J Pathol 196: 335-342, 2002.

47. Shi Y and Tokunaga O: Chlamydia pneumoniae (C. pneumoniae) infection upregulates atherosclerosis-related gene expression in human umbilical vein endothelial cells (HUVECs). Atherosclerosis 177: 245-253, 2004.

48. Anikhovskaia IA, Kubatiev AA and Iakovlev Mlu: Endotoxin theory of atherosclerosis. Fiziol Cheloveka 41: 106-116, 2015 (In Russian).

49. Wort SJ and Evans TW: The role of the endothelium in modulating vascular control in sepsis and related conditions. Br Med Bull 55: 30-48, 1999.

50. Hennessy EJ,Sheedy FJ, Santamaria D, Barbacid M and O'Neill LA: Toll-like receptor-4 (TLR4) down-regulates microRNA-107, increasing macrophage adhesion via cyclin-dependent kinase 6. J Biol Chem 286: 25531-25539, 2011.

51. Kumar V and Mahato RI: Delivery and targeting of miRNAs for treating liver fibrosis. Pharm Res 32: 341-361, 2015. 